\title{
Association between life-course socio-economic status and prevalence of cardio-metabolic risk factors in five middle-income countries
}

\author{
Kemi Ogunsina ${ }^{1}$, \\ Daniel T Dibaba ${ }^{2,3}$, \\ Tomi Akinyemiju ${ }^{2,3}$ \\ ${ }^{1}$ Department of Epidemiology, University \\ of Miami, Miami, Florida, USA \\ ${ }^{2}$ Department of Epidemiology, University \\ of Kentucky, Lexington, Kentucky,USA \\ ${ }^{3}$ Markey Cancer Center, University of \\ Kentucky, Lexington, Kentucky, USA
}

Background The burden of non-communicable diseases has increased rapidly in low- and middle-income countries. Past studies have reported an association between socioeconomic status (SES) and cardio-metabolic risk factors, but most have focused on upper income countries. The purpose of this study is to examine the association between SES over the lifecourse and the burden of cardio-metabolic risk factors in middle-income countries.

Methods A total of 38297 adults from China, Mexico, India, South Africa and Russia were included in this cross-sectional study. Life-course SES was defined based on maternal and participant education, and data on blood pressure, body mass index (BMI), self-reported diabetes and hypertension were obtained by trained interviewers. Descriptive, age standardized and multivariable adjusted analyses were conducted using survey weighted statistical procedures in SAS 9.4 (SAS Institute, Cary, NC, USA).

Results Although 14\% of men and 12\% of women had current hypertension based on blood pressure measurements, only $2 \%$ of men and $4 \%$ of women were aware of their hypertensive status. Men with stable high life-course SES had higher odds of being overweight/obese (odds ratio $\mathrm{OR}=2.01,95 \%$ confidence interval $(\mathrm{CI})=1.30-3.10)$, diabetic $(\mathrm{OR}=4.82$, $95 \% \mathrm{CI}=2.07-11.2)$ and hypertensive based on self-report ( $\mathrm{OR}=3.42,95 \%$ $\mathrm{CI}=1.85-6.32)$ compared to men of low life-course SES. Among women, the odds of being overweight/obese were significantly higher among women with high life-course SES $(\mathrm{OR}=1.50,95 \% \mathrm{CI}=1.08-2.08)$.

Conclusions Higher life-course SES for both men and women was associated with increased odds of overweight/ obesity, and additionally diabetes and hypertension for men in middle income countries.

\section{Correspondence to:}

Tomi Akinyemiju, PhD Department of Epidemiology University of Kentucky College of Public Health

111 Washington Ave. Lexington, KY 40536-0003

United States of America

tomiakin@uky.edu
Cardio-metabolic risk factors such as hypertension, obesity and diabetes are associated with increasing burden of morbidity and mortality globally [1,2] . Chronic diseases associated with these risk factors were responsible for approximately $60 \%$ of all deaths globally in $2008[3,4]$, an estimate that is projected to increase to $80 \%$ by the year 2020[5]. Although the majority of studies evaluating cardio-metabolic risk factors have traditionally focused on upper income countries, lower- and middle-income country (LMICs) populations are increasingly being identified as highly susceptible due to the epidemiologic transition. The transition is characterized by rapid population growth, ageing, transitions from traditional diets high in fruits and leafy vegetables and high physical activ- 
ity levels to increased consumption of calorie-dense meals and sedentary lifestyles, leading to increasing rates of obesity [6]. Cardiovascular diseases, cancers, and chronic respiratory diseases account for over two-thirds of deaths in LMICs, and are closely linked with cardio-metabolic risk factors such as diabetes, hypertension and obesity [7].

Socioeconomic status (SES) at both individual [8], parental [9] and across the life-course [8] has been associated with the development of cardio-metabolic risk factors in many upper income countries [10, 11]. In addition, individual and parental SES has been found to predict health behavior and access to preventive health care $[12,13]$. In high-income countries, lower individual or parental SES has been found to increase the risk of obesity, diabetes and hypertension [10,12,14]; rural residents are also found to have higher risks of cardio-metabolic syndrome compared with urban residents [15]. In LMICs however, fewer studies have examined the association between SES and cardio-metabolic risk factors. SES assessed over the life-course may provide important clues into the accumulation of risk that occurs from childhood, particularly health habits that may predispose individuals to unhealthy diets and low physical activity, leading to increased risk of chronic diseases. Interventions to reduce the burden of chronic diseases in LMICs may benefit from a focus on early life critical periods for both prevention and elimination of cardio-metabolic risk factors. Here, we examine the prevalence of cardio-metabolic risk factors in 5 middle-income countries and their association with life-course SES.

\section{METHODS}

\section{Data source and study population}

The data used for this analysis was obtained from wave one of the World Health Organization (WHO) Study on Global Ageing and Adult Health (SAGE) conducted between 2007 and 2010. The SAGE study was designed as a longitudinal study aimed to assess well-being, quality of life, and access to health care for individuals in developing countries, and obtained data across populations in six different countries; China, Ghana, India, Mexico, South Africa and Russia. A multistage cluster sampling study design was utilized to create nationally representative cohorts from each country. Survey instruments were adapted and modified from the World Health Survey (WHS) and used for individual and household interviews. Trained field staff obtained measurements of blood pressure, height and weight of participants, and a detailed questionnaire translated to the local languages were administered. More information on the SAGE study can be obtained through the WHO SAGE website: (http://www.who.int/healthinfo/sage/cohorts/ en/). The current analysis focused on one country from each continent represented in SAGE and included 38297 participants ages 18 and older from the nationally representative samples of five middle-income countries - China, India, Mexico, Russia and South Africa.

\section{Cardio-metabolic risk factors}

Cardio-metabolic risk factors assessed included body mass index (BMI), current hypertension, history of hypertension, and history of diabetes. BMI was calculated based on measurement of height and weight and categorized into normal weight (BMI $18.5-24.9 \mathrm{~kg} / \mathrm{m}^{2}$ ) and overweight/obese (BMI $\geq 25.0 \mathrm{~kg} / \mathrm{m}^{2}$ ). Current hypertension was based on the average of 3 blood pressure readings obtained by trained field personnel, and classified as present if average blood pressure reading was $\geq 140 / 90 \mathrm{~mm} \mathrm{Hg}$. History of hypertension was defined based on self-reported ever diagnosed of hypertension, use of anti-hypertensive medications or diet modification to control hypertension. History of diabetes was defined based on self-reported ever diagnosed of diabetes and the use of anti-diabetes medication or dietary control of diabetes.

\section{Life-course socio-economic status}

Life-course SES was categorized based on maternal and participant educational attainment as follows: stable-high if both mother and participant had $\geq$ primary school education, increasing if mother had $<$ primary school education while participant had $\geq$ primary, declining if mother had $\geq$ primary and participant had < primary school education, and stable-low if both mother and participant had < primary school education. Studies have shown that parental SES, in particular maternal SES, is positively associated with offspring's overall health [8]. For instance, highly educated mothers have greater decision making powers on the health and welfare of their child, they are more knowledgeable about disease prevention methods [16]. Highly educated mothers are less likely to smoke or consume excessive alcohol, and more likely to complete prenatal check-ups [17]. These positive early life influences have also been shown to extend 
to adulthood, with daughters of higher SES mothers less likely to be obese compared with daughters of lower SES mothers [18].

\section{Covariates}

Other covariates included in the analysis were age, gender, marital status, rural/urban residence, country, and current health status. All covariates were analyzed as categorical variables, selected based on the known contribution to the outcome in the presence of other variables.

\section{Statistical analysis}

Survey-weighted frequencies and percentages and $\chi^{2}$ tests were used to compare the distribution of baseline demographics and cardio-metabolic risk factors by country. Prevalence of cardio-metabolic risk factors were standardized by age using the world population by age and sex from United States Census Bureau International Data Base [19], using standard age categories: 18-24, 25-39, 40- 64, $\geq 65$ years. Survey weighted multivariable logistic regression models were used to determine the association between life-course SES and cardio-metabolic risk factors. All regression models adjusted for age, marital status, rural/urban residence, health status, and country to compute adjusted estimates for the main predictor. For all analyses, $P$-values $\leq 0.05$ were considered statistically significant. All study participants and individual weights were retained in the analysis to ensure valid inferences and representativeness of results to the source population. Although less than 1 percent of study participants had missing data on the main covariates of interest, participants with missing data had higher mean diastolic blood pressure (DBP) $(P<0.001)$, systolic blood pressure $(\mathrm{SBP})(P<0.001)$ and BMI $(P<0.001)$ than participants with complete data but there was no difference in type 2 diabetes status $(P=0.763)$. All statistical analyses were conducted in SAS 9.4 (SAS Institute Inc. NC, USA) using survey weights, strata and cluster variables provided with the SAGE data set, thus enabling study results to be generalizable to the entire population of included countries.

\section{RESULTS}

\section{Participant characteristics}

A total of 38297 participants were included in this analysis: 16014 men and 22283 women ages 18 years and older (Table 1); of these, 14991 participants were from China, 12198 from India, 2733 from Mexico, 4223 from South Africa and 4152 from Russia. The majority of study participants were married (59\%) and more than $45 \%$ of both male and female participants in Russia, South Africa, and China had completed a secondary school education; however, there were large gender disparities in completion of secondary school education in India and Mexico. For instance, India had the highest proportion of women with no formal education (55\%), and the largest gender disparity in education of $28 \%$. About $84 \%$ of men and $82 \%$ of women in Russia reported stable high life-course SES, compared with 19\% of men and 33\% of women in South Africa, 29\% of men and 16\% of women in Mexico, $20 \%$ of men and 19\% of women in China, and 12\% of both genders in India. Over $50 \%$ of men and women in China reported declining SES, while 34\% of women and 45\% of men in South Africa reported stable low life-course SES.

\section{Prevalence of cardio-metabolic risk factors}

Mexico had the highest prevalence of overweight/obesity, with age standardized rate of 841 and 699 per 1000 population for men and women respectively, followed by South Africa with prevalence of 676 and 686 per 1000 for men and women respectively (Table 2). The lowest rate of overweight/obesity was observed in India, with a prevalence of 134 and 232 per 1000 for men and women, respectively. The age standardized prevalence of current hypertension was highest in South Africa, at 348 and 269 per 1000 for men and women respectively, followed by China, with 168 per 1000 for men. India had the lowest age standardized prevalence of current hypertension, 80 and 84 per 1000 men and women respectively. Across countries, the prevalence of self-reported hypertension was significantly lower compared with current hypertension, except among Chinese men (331 per 1000 self-reported hypertension vs 168 per 1000 current hypertension) and Russian women (182 per 1000 self-reported hypertension vs 149 per 1000 current hypertension). The prevalence of diabetes was highest among Russian women at 132 per 1000 women, followed by Mexican men and women at 84 and 89 per 1000 respectively, and lowest among 
Table 1. Socio-demographic and life-course SES characteristics of SAGE participants

\begin{tabular}{|c|c|c|c|c|c|c|c|c|c|c|}
\hline \multirow[t]{2}{*}{ TotAL ( $(\mathrm{N}=38297)$} & \multicolumn{2}{|c|}{ Cнпин ( $(n=14991)$} & \multicolumn{2}{|c|}{ Mexico (n = 2733) } & \multicolumn{2}{|c|}{ INDIA ( } & \multicolumn{2}{|c|}{ South Africa ( $=4223$ ) } & \multicolumn{2}{|c|}{ Russia ( $(=4152$ ) } \\
\hline & $\begin{array}{c}\text { Male } \\
(\mathrm{n}=6989)\end{array}$ & $\begin{array}{c}\text { Female } \\
(\mathbf{n}=8002)\end{array}$ & $\begin{array}{c}\text { Male } \\
(n=1044)\end{array}$ & $\begin{array}{c}\text { Female } \\
(\mathrm{n}=1689)\end{array}$ & $\begin{array}{c}\text { Male } \\
(n=4709)\end{array}$ & $\begin{array}{c}\text { Female } \\
(\mathrm{n}=7489)\end{array}$ & $\begin{array}{c}\text { Male } \\
(n=1796)\end{array}$ & $\begin{array}{c}\text { Female } \\
(\mathrm{n}=2427)\end{array}$ & $\begin{array}{c}\text { Male } \\
(n=1476)\end{array}$ & $\begin{array}{c}\text { Female } \\
(\mathrm{n}=2676)\end{array}$ \\
\hline \multicolumn{11}{|l|}{ Age group: } \\
\hline $18-24$ & $49(5.4)$ & $42(3.3)$ & $5(6.1)$ & $9(0.9)$ & $146(10.2)$ & $964(18.7)$ & $28(13.5)$ & $33(12.1)$ & $24(15.7)$ & $19(4.0)$ \\
\hline $25-39$ & $267(23.1)$ & $371(27.7)$ & $71(46.3)$ & $155(50.6)$ & $515(34.3)$ & $1961(36.1)$ & $69(32.0)$ & $100(31.9)$ & $76(28.2)$ & $110(27.6)$ \\
\hline $40-64$ & $4121(62.1)$ & $4730(58.3)$ & $422(38.2)$ & $704(38.0)$ & $2612(46.6)$ & 3357 (36.6) & $1118(47.8)$ & $1390(46.8)$ & $792(43.4)$ & $1270(46.2)$ \\
\hline$>65$ & $2552(9.0)$ & $2859(10.6)$ & $546(9.3)$ & $821(10.5)$ & $1436(8.9)$ & $1225(8.6)$ & $581(6.7)$ & $904(9.2)$ & $584(12.7)$ & $1277(22.2)$ \\
\hline$P$-value & \multicolumn{2}{|c|}{0.018} & \multicolumn{2}{|c|}{0.048} & \multicolumn{2}{|c|}{$<0.0001$} & \multicolumn{2}{|c|}{0.896} & \multicolumn{2}{|c|}{0.013} \\
\hline
\end{tabular}

Marital status:

\begin{tabular}{lllllllllll}
\hline Married & $5500(89.8)$ & $5730(90.0)$ & $713(73.0)$ & $803(66.4)$ & $2436(86.9)$ & $3297(80.1)$ & $1325(70.7)$ & $800(37.7)$ & $1068(69.7)$ & $1085(54.0)$ \\
\hline Nerer & & $147(6.4)$ & $79(3.2)$ & $60(22.6)$ & $168(18.9)$ & $113(10.1)$ & $239(7.2)$ & $199(24.2)$ & $414(33.5)$ & $49(18.5)$
\end{tabular}

\begin{tabular}{lllllllllll}
\hline Never married & $147(6.4)$ & $79(3.2)$ & $60(22.6)$ & $168(18.9)$ & $113(10.1)$ & $239(7.2)$ & $199(24.2)$ & $414(33.5)$ & $49(18.5)$ & $101(7.9)$ \\
\hline
\end{tabular}

\begin{tabular}{|c|c|c|c|c|c|c|c|c|c|}
\hline Widow/divorced & $517(3.8)$ & $1343(6.7)$ & 137 (3.9) & $531(14.6)$ & $208(2.9)$ & $893(12.7)$ & $250(5.1)$ & $1015(28.9)$ & \\
\hline
\end{tabular}

\begin{tabular}{llllll}
\hline$P$-value & $<0.0001$ & 0.037 & $<0.0001$ & $<0.0001$ & $<0.0001<$
\end{tabular}

Highest education:

\begin{tabular}{lllllllllll}
\hline No formal & $1786(14.5)$ & $3384(24.8)$ & $474(23.5)$ & $794(29.4)$ & $983(27.2)$ & $2666(54.7)$ & $585(26.1)$ & $962(20.7)$ & $14(0.3)$ & $82(1.2)$ \\
\hline
\end{tabular}

\begin{tabular}{lllllllllll}
\hline Primary school & $1437(20.7)$ & $1215(17.1)$ & $210(20.9)$ & $322(30.8)$ & $461(15.6)$ & $667(16.8)$ & $288(11.7)$ & $461(15.4)$ & $85(2.1)$ & $172(2.3)$ \\
\hline Secol
\end{tabular}

\begin{tabular}{lllllllllll}
\hline Secondary school & $2530(55.8)$ & $2288(49.7)$ & $146(42.4)$ & $200(29.4)$ & $971(41.3)$ & $897(23.6)$ & $299(56.9)$ & $457(54.4)$ & $941(71.6)$ & $1665(77.1)$ \\
\hline
\end{tabular}

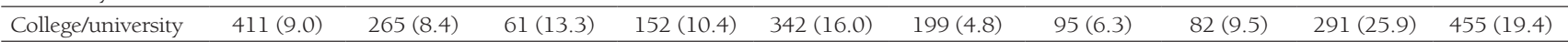

$\begin{array}{llllll}P \text {-value } & 0.118 & 0.325 & <0.0001 & 0.757 & 0.585\end{array}$

\begin{tabular}{|c|c|c|c|c|c|c|c|c|c|c|}
\hline \multicolumn{11}{|c|}{ Employment status: } \\
\hline Unemployed & $2474(17.6)$ & $3400(27.5)$ & $272(11.5)$ & $332(16.2)$ & $735(10.5)$ & $823(15.6)$ & $838(29.9)$ & $1329(39.7)$ & $719(23.8)$ & $1539(35.5)$ \\
\hline Private sector & $451(16.6)$ & $242(9.0)$ & $131(20.3)$ & $65(7.6)$ & 199 (9.9) & $152(3.6)$ & $342(28.5)$ & $212(11.6)$ & $140(20.6)$ & $120(4.2)$ \\
\hline Public sector & $671(17.2)$ & $456(15.5)$ & $54(10.4)$ & $37(3.6)$ & $235(8.5)$ & $95(2.0)$ & $99(6.9)$ & $98(14.9)$ & $408(42.4)$ & $636(51.1)$ \\
\hline Self-employed & $2568(48.6)$ & $3054(48.0)$ & $453(57.9)$ & $1068(72.6)$ & $1588(71.1)$ & $3359(78.6)$ & $345(34.7)$ & $590(33.9)$ & $65(13.2)$ & $79(4.6)$ \\
\hline$P$-value & \multicolumn{2}{|c|}{0.084} & \multicolumn{2}{|c|}{0.613} & \multicolumn{2}{|c|}{$<0.0001$} & \multicolumn{2}{|c|}{0.046} & \multicolumn{2}{|c|}{$<0.0001$} \\
\hline \multicolumn{11}{|l|}{ Health status: } \\
\hline Good & $2515(56.3)$ & $2415(49.6)$ & $402(66.0)$ & $568(47.8)$ & $1217(58.8)$ & $1777(48.5)$ & $763(71.4)$ & $867(56.6)$ & $296(46.0)$ & $304(37.7)$ \\
\hline Moderate & $2624(33.2)$ & $3264(38.0)$ & $426(30.0)$ & $756(41.4)$ & $1211(33.5)$ & $2082(41.3)$ & $634(21.3)$ & $1030(32.3)$ & $772(45.5)$ & $1426(50.0)$ \\
\hline $\mathrm{Bad}$ & $1025(10.5)$ & $1473(12.6)$ & $82(4.0)$ & $178(10.7)$ & $329(7.6)$ & $570(10.2)$ & $227(7.4)$ & $332(11.0)$ & $264(8.4)$ & $644(12.4)$ \\
\hline$P$-value & \multicolumn{2}{|c|}{0.003} & \multicolumn{2}{|c|}{0.056} & \multicolumn{2}{|c|}{$<0.0001$} & \multicolumn{2}{|c|}{0.181} & \multicolumn{2}{|c|}{0.475} \\
\hline \multicolumn{11}{|c|}{ Life-course SES:* } \\
\hline Stable high & $548(19.7)$ & $617(18.2)$ & $96(28.7)$ & $140(15.6)$ & $211(11.6)$ & 475 (12.4) & $192(18.8)$ & $292(33.4)$ & 835 (83.9) & $1483(81.5)$ \\
\hline Increasing & $10(0.2)$ & $26(0.2)$ & $10(0.3)$ & $16(1.5)$ & $7(0.5)$ & $60(1.4)$ & $33(1.4)$ & $42(1.2)$ & $3(0.1)$ & $10(0.1)$ \\
\hline Declining & $3830(65.7)$ & $3151(56.9)$ & $321(47.2)$ & $534(54.5)$ & $1563(61.2)$ & $1288(32.8)$ & $490(34.2)$ & $708(31.4)$ & $482(15.8)$ & $809(17.3)$ \\
\hline Stable low & $1776(14.3)$ & $3358(24.6)$ & $483(23.7)$ & $812(28.4)$ & $976(26.7)$ & $2606(53.3)$ & 909 (45.6) & $1187(34.0)$ & $12(0.3)$ & $72(1.0)$ \\
\hline$P$-value & \multicolumn{2}{|c|}{0.066} & \multicolumn{2}{|c|}{0.073} & \multicolumn{2}{|c|}{$<0.0001$} & \multicolumn{2}{|c|}{0.528} & \multicolumn{2}{|c|}{0.023} \\
\hline
\end{tabular}

SES - socio-economic status, SAGE - Study on Global Ageing and Adult Health

* Based on maternal and participants level of education. $P$-values testing for significant differences between males and females within each country.

Table 2. Distribution and age standardized prevalence of cardio-metabolic risk factors per 1000 population

\begin{tabular}{|c|c|c|c|c|c|c|c|c|c|c|}
\hline & \multicolumn{2}{|c|}{ China } & \multicolumn{2}{|c|}{ INDIA } & \multicolumn{2}{|c|}{ Mexico } & \multicolumn{2}{|c|}{ South Africa } & \multicolumn{2}{|c|}{ Russia } \\
\hline & $\mathbf{N}(\%)$ & Rate & $\mathbf{N}(\%)$ & Rate & $\mathbf{N}(\%)$ & Rate & $\mathbf{N}(\%)$ & Rate & $\mathbf{N}(\%)$ & Rate \\
\hline \multicolumn{11}{|c|}{ Overweight/obese: } \\
\hline Male & $1802(30.3)$ & 286.2 & $442(16.3)$ & 134.0 & $572(71.0)$ & 841.4 & $999(67.0)$ & 676.0 & $869(69.7)$ & 562.4 \\
\hline Female & $2627(37.9)$ & 420.6 & $1101(25.2)$ & 232.1 & $1044(77.2)$ & 699.4 & $1555(77.0)$ & 686.2 & $1750(77.6)$ & 477.6 \\
\hline \multicolumn{11}{|c|}{ Current hypertension* } \\
\hline Male & $1590(23.5)$ & 168.2 & $512(11.0)$ & 79.6 & $163(17.6)$ & 123.7 & $724(43.8)$ & 347.7 & $363(26.4)$ & 124.9 \\
\hline Female & $1674(21.6)$ & 114.3 & $590(8.0)$ & 84.2 & $215(14.1)$ & 122.0 & $1014(46.0)$ & 268.8 & $818(32.2)$ & 148.8 \\
\hline \multicolumn{11}{|c|}{ Hypertension - self-reported $\dagger$} \\
\hline Male & $1179(18.1)$ & 330.6 & $265(6.2)$ & 44.6 & $154(17.5)$ & 61.8 & $300(19.1)$ & 55.5 & $386(28.4)$ & 119.2 \\
\hline Female & $1608(21.5)$ & 89.1 & $395(5.8)$ & 65.0 & $403(27.7)$ & 99.8 & $586(27.9)$ & 94.2 & $1178(47.0)$ & 182.4 \\
\hline \multicolumn{11}{|c|}{ Diabetes: $\ddagger$} \\
\hline Male & $299(4.6)$ & 18.2 & $153(3.6)$ & 22.1 & $116(13.2)$ & 84.4 & $103(6.6)$ & 24.0 & $47(3.5)$ & 8.6 \\
\hline Female & $398(5.3)$ & 23.0 & 131 (1.9) & 16.7 & $239(16.4)$ & 88.6 & $178(8.5)$ & 30.7 & $194(7.7)$ & 132.1 \\
\hline
\end{tabular}

*Current hypertension was defined based on mean of three systolic blood pressure $\geq 140 \mathrm{~mm} \mathrm{Hg}$ or diastolic blood pressure $\geq 90 \mathrm{~mm} \mathrm{Hg}$.

$\uparrow$ Self-reported hypertension was defined as self-reported previously diagnosed hypertension and/or medication use.

₹Diabetes was defined as self-reported diagnosed diabetes on dietary management and/or medication use. 
Russian men (9/1000), Indian women (17/1000) and Chinese men (18/1000). Overall, 13\% of the participants had current hypertension but only $24 \%$ of them were aware that they had hypertension. Although $14 \%$ of men and $12 \%$ of women overall had hypertension based on blood pressure measurements, only $2 \%$ of men and $4 \%$ of women were aware of their hypertension status (data not shown).

\section{Life-course SES and cardio-metabolic risk factors}

Men with stable high $(\mathrm{OR}=2.01,95 \%$ confidence interval $(\mathrm{CI})=1.30-3.10)$ or declining $\mathrm{SES}(\mathrm{OR}=1.79$, 95\% CI =1.34-2.40) had higher odds of overweight/obesity compared with men in stable low SES, while women with stable high $(\mathrm{OR}=1.50,95 \% \mathrm{CI}=1.08-2.08)$, increasing $(\mathrm{OR}=2.10,95 \% \mathrm{CI}=1.10-3.96)$ or declining $(\mathrm{OR}=1.38,95 \% \mathrm{CI}=1.18-1.71)$ life-course SES had higher odds of overweight/obesity compared with women with stable low SES (Table 3). In addition, the odds of diabetes were higher among men with stable high $(\mathrm{OR}=4.82,95 \% \mathrm{CI}=2.07-11.2)$ and declining $(\mathrm{OR}=3.12,95 \% \mathrm{CI}=1.93-5.02)$ life-course SES compared with stable low life-course SES, but there was no significant association among women. There was a 3-fold higher odds of self-reported hypertension associated with stable high compared with stable low life-course SES ( $\mathrm{OR}=3.42,95 \% \mathrm{CI}=1.85-6.32)$, but this association was only observed among men. Men residing in urban vs rural areas also had increased odds of overweight/obesity $(\mathrm{OR}=1.33,95 \% \mathrm{CI}=1.03-1.70)$ as well as diabetes and history of hypertension, although these estimates were not statistically significant. The prevalence of each cardio-metabolic risk factor by life-course SES for each country is presented in Figure 1.

\section{Distribution of cardio-metabolic risk factors by SES in China}

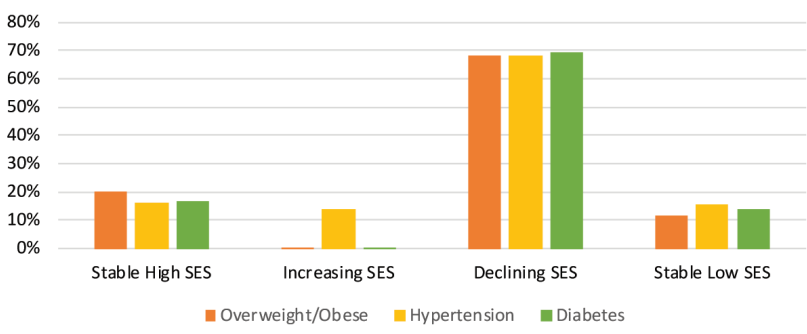

\section{Distribution of cardio-metabolic risk factors by SES in Mexico}

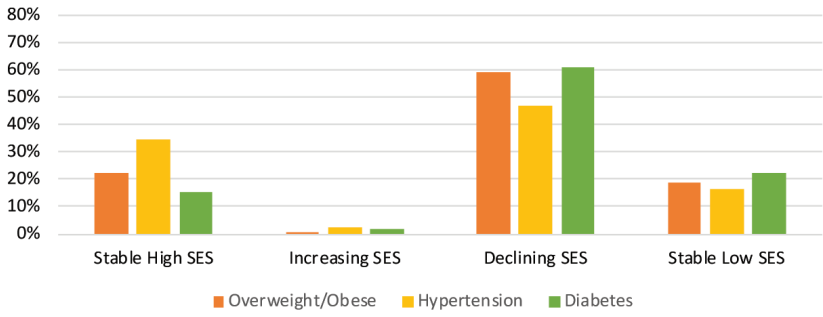

\section{Distribution of cardio-metabolic risk factors by SES in South Africa}

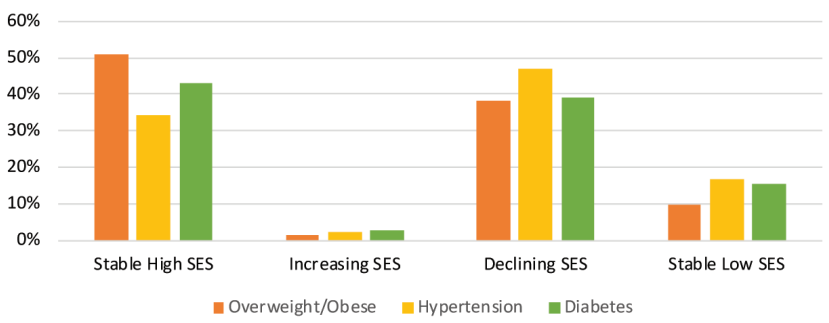

\section{Distribution of cardio-metabolic risk factors by SES in India}

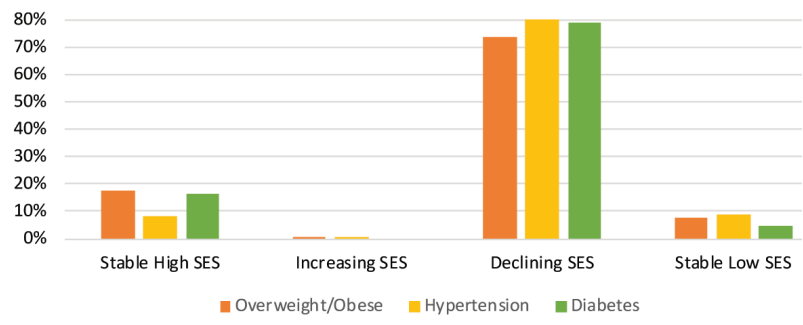

Distribution of cardio-metabolic risk factors by SES in Russia

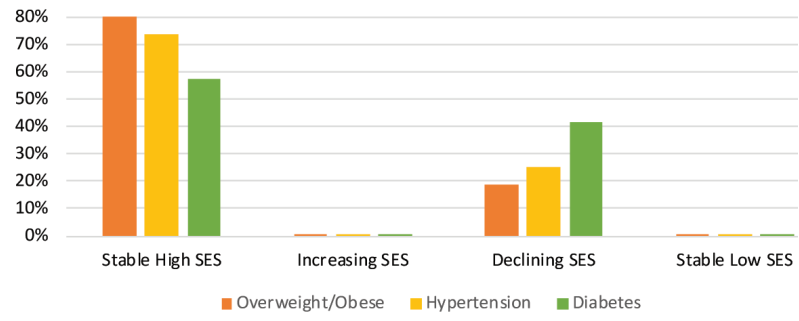

Figure 1. Prevalence of cardio-metabolic risk factors by life-course SES across countries. 
Table 3. Association between life-course SES and cardio-metabolic risk factors by gender*

\begin{tabular}{|c|c|c|c|c|c|c|c|c|}
\hline & \multicolumn{4}{|c|}{ MALE N $=12787$} & \multicolumn{4}{|c|}{ Female $N=17686$} \\
\hline & $\begin{array}{c}\text { Overweight/ } \\
\text { obese } \\
\text { OR }(95 \% \mathrm{CI})\end{array}$ & $\begin{array}{c}\text { Diabetes } \\
\text { OR }(95 \% \mathrm{CI})\end{array}$ & $\begin{array}{l}\text { Self-reported } \\
\text { hypertension } \\
\text { OR }(95 \% \mathrm{CI})\end{array}$ & $\begin{array}{c}\text { Current } \\
\text { hypertension } \\
\text { OR }(95 \% \mathrm{CI})\end{array}$ & $\begin{array}{c}\text { Overweight/ } \\
\text { obese } \\
\text { OR }(95 \% \text { CI })\end{array}$ & $\begin{array}{l}\text { Diabetes OR } \\
(95 \% \mathrm{CI})\end{array}$ & $\begin{array}{l}\text { Self-reported } \\
\text { hypertension } \\
\text { OR }(95 \% \mathrm{CI})\end{array}$ & $\begin{array}{c}\text { Current } \\
\text { hypertension } \\
\text { OR }(95 \% \mathrm{CI})\end{array}$ \\
\hline \multicolumn{9}{|c|}{ Life-course SES: $\dagger$} \\
\hline Stable high & $2.01(1.30-3.10)$ & $4.82(2.07-11.2)$ & $3.42(1.85-6.32)$ & $1.17(0.72-1.92)$ & $1.50(1.08-2.08)$ & $0.81(0.34-1.91)$ & $0.83(0.54-1.27)$ & $0.78(0.52-1.18)$ \\
\hline Increasing & $0.96(0.34-2.68)$ & $1.57(0.28-8.78)$ & $0.90(0.23-3.64)$ & $0.42(0.15-1.18)$ & $2.10(1.10-3.96)$ & $0.85(0.30-2.43)$ & $0.62(0.34-1.13)$ & $1.35(0.55-3.34)$ \\
\hline Declining & $1.79(1.34-2.40)$ & $3.12(1.93-5.02)$ & $1.33(0.99-1.81)$ & $0.98(0.71-1.35)$ & $1.38(1.18-1.71)$ & $1.00(0.59-1.70)$ & $0.99(0.75-1.33)$ & $0.92(0.71-1.19)$ \\
\hline Stable low & Ref & Ref & Ref & Ref & Ref & Ref & Ref & Ref \\
\hline \multicolumn{9}{|l|}{ Age } \\
\hline $18-24$ & $0.51(0.21-1.28)$ & $0.09(0.03-0.29)$ & $0.01(0.00-0.03)$ & $0.20(0.08-0.50)$ & $0.23(0.14-0.37)$ & - & $0.01(0.00-0.06)$ & $0.09(0.03-0.25)$ \\
\hline $25-39$ & $0.64(0.45-0.90)$ & $0.06(0.02-0.18)$ & $0.04(0.02-0.10)$ & $0.28(0.19-0.40)$ & $0.31(0.22-0.44)$ & 0.06 & $0.02(0.01-0.04)$ & $0.14(0.10-0.21)$ \\
\hline $40-64$ & $1.01(0.82-1.25)$ & $0.30(0.21-0.42)$ & $0.21(0.16-0.28)$ & $0.63(0.51-0.78)$ & $0.73(0.59-0.89)$ & $0.42(0.27-0.68)$ & $0.26(0.21-0.32)$ & $0.51(0.41-0.62)$ \\
\hline$\geq 65$ & Ref & Ref & Ref & Ref & Ref & Ref & Ref & Ref \\
\hline \multicolumn{9}{|l|}{ Marital status: } \\
\hline Married & $1.94(0.94-4.00)$ & $1.84(0.69-4.93)$ & $1.73(0.66-4.51)$ & $1.46(0.83-2.57)$ & $2.00(1.19-3.31)$ & $1.23(0.63-2.40)$ & $0.99(0.52-1.88)$ & $1.02(0.58-1.80)$ \\
\hline Wid/divorced & $1.50(0.62-3.63)$ & $2.02(0.68-6.06)$ & $1.97(0.54-7.18)$ & $1.15(0.63-2.13)$ & $1.21(0.68-2.17)$ & $1.46(0.74-2.88)$ & $0.77(0.41-1.45)$ & $1.12(0.62-2.05)$ \\
\hline Never married & Ref & Ref & Ref & Ref & Ref & Ref & Ref & Ref \\
\hline \multicolumn{9}{|l|}{ Residence: } \\
\hline Urban & $1.33(1.03-1.70)$ & $1.62(0.96-2.76)$ & $1.49(0.93-2.36)$ & $0.91(0.68-1.23)$ & $0.86(0.71-1.04)$ & $1.60(0.99-2.59)$ & $1.28(0.95-1.74)$ & $0.63(0.48-0.82)$ \\
\hline Rural & Ref & Ref & Ref & Ref & Ref & Ref & Ref & Ref \\
\hline \multicolumn{9}{|l|}{ Country: } \\
\hline China & $0.23(0.14-0.39)$ & $0.74(0.35-1.57)$ & $1.08(0.68-1.70)$ & $0.32(0.19-0.55)$ & $0.13(0.08-1.04)$ & $0.78(0.48-1.27)$ & $0.65(0.43-0.98)$ & $0.30(0.21-0.44)$ \\
\hline India & $0.11(0.06-0.18)$ & $1.13(0.48-2.67)$ & $0.74(0.44-1.23)$ & $0.14(0.08-0.25)$ & $0.10(0.06-0.17)$ & $0.63(0.38-1.04)$ & $0.44(0.29-0.67)$ & $0.17(0.12-0.26)$ \\
\hline Mexico & $3.09(1.45-6.58)$ & $4.52(1.65-12.4)$ & $0.71(0.40-1.27)$ & $0.26(0.13-0.52)$ & $1.08(0.57-2.06)$ & $3.30(1.81-6.02)$ & $0.74(0.46-1.18)$ & $0.24(0.14-0.41)$ \\
\hline Russia & $0.64(0.31-1.34)$ & $0.20(0.08-0.49)$ & $1.10(0.58-2.12)$ & $0.24(0.13-0.47)$ & $0.39(0.20-0.77)$ & $0.92(0.44-1.91)$ & $2.10(1.34-3.29)$ & $0.56(0.36-0.89)$ \\
\hline South Africa & Ref & Ref & Ref & Ref & Ref & Ref & Ref & Ref \\
\hline
\end{tabular}

SES - socio-economic status, OR - odds ratio, CI - confidence interval

*OR and 95\% CI reported adjusted for age, marital status, country, rural/urban residence, health status and socioeconomic status. Statistically significant ORs are bolded.

$\nmid$ Life-course SES variable was based on maternal and participant level of education.

\section{DISCUSSION}

In a nationally representative sample of adults in five middle-income countries (China, South Africa, India, Russia and Mexico), we observed that the population distribution of stable high life-course SES ranged from 18\% in China to over 80\% in Russia. In addition, the highest age-standardized rates of overweight/ obesity, history of hypertension, and diabetes were observed in Mexico, South Africa, Russia, respectively. Across the included countries, men with stable high life-course SES had two to four times higher odds of being overweight/obese, diabetic and hypertensive compared with men of stable low life-course SES. While women with stable high, declining and increasing life-course SES had up to a 2-fold increased odds of overweight/obesity compared with stable low life-course SES. We also observed that a significant proportion (13\%) of participants were hypertensive based on blood pressure measurements obtained during in-person interviews, but $76 \%$ them had no prior knowledge of having hypertension. The largest disparity between self-reported history of hypertension and current hypertension was observed in South Africa where only $6 \%$ of men with hypertension were aware that they had hypertension or high blood pressure, whereas 38\% had hypertension based on blood pressure measures.

The associations observed in this study are consistent with existing studies showing that in lower and middle income countries, higher SES individuals have a higher prevalence of obesity, diabetes and hypertension compared with lower SES individuals, while in high-income countries, the association is typically inverse [20-22]. Few studies have evaluated SES over the life-course in lower and middle-income countries, an area that deserves greater attention given the rapid economic development and effects of globalization in these countries. Life-course SES is hypothesized to influence adult health through the accumulation of different SES experiences throughout life; increasing life-course SES indicates that higher adult SES may attenuate the negative effects of early life low SES, while decreasing life-course SES indi- 
cates that lower adult SES may have a negative influence on adult health despite relatively higher SES in early life [23]. Low SES across the life-course is associated with increased prevalence of obesity, diabetes and hypertension [18] in developed countries; however, results on the influence of increasing and declining life-course SES have been mixed. In the countries evaluated in this study, the association between life-course SES and cardio-metabolic risk factors was most consistent for overweight/obesity among both men and women, with significantly higher odds for those with stable high, declining or increasing lifecourse SES. History of diabetes and hypertension were also significantly higher among men with stable high life-course SES, but not among women. Other studies from developing countries have reported conflicting results; some studies report a direct or no association between SES and cardio-metabolic risk factors [24-27], while other studies observed that the association varies by geographic location [28], or on the measures of SES used [29]. These differences may be due to between country heterogeneity in the level of economic development, ie some middle-income countries are likely more economically developed than others, eg despite both being classified as middle-income countries, almost $20 \%$ of women in Russia had completed college/university education, compared with 5\% in India. It may also be due to within country differences in the association between SES and lifestyle based on social norms such as cultural standards of ideal body weight.

The potential mechanisms explaining the association between SES and cardio-metabolic risk factors in the studied countries may include socially patterned behavioral risk factors such as alcohol use, dietary quality and physical activity [20]. Alcohol consumption of greater than 7 drinks per week has been associated with obesity especially in men [30], and increased alcohol consumption is associated with higher income. Dietary patterns in many middle-income countries have also changed dramatically as a result of economic development and globalization. The concentration of overweight/obesity among higher SES groups suggest that lack of affordability of the relatively new dietary items may act as a protective factor against increasing obesity rates among lower SES groups. A third potential mechanism involves access to health care- higher life-course SES was associated with increased odds of self-reported hypertension, but not interviewer measured hypertension based on high blood pressure. In addition, only a minority of adults who were hypertensive based on blood pressure measurements were aware of their hypertensive status. This is likely due to improved access to health care among higher SES groups, leading to clinical diagnosis, and potentially treatment, for hypertension, and under-estimation of hypertension prevalence in lower SES groups [31-33]. A separate study in China also observed that only $28 \%$ of diabetic patients were aware of their diabetic status, and only 23\% had received treatment [26]. Studies in the US show that higher SES individuals are often the first to adopt health measures to enhance their health and overall well-being by taking advantage of known protective health factors, thus explaining the SES gradient observed in health [34].

Cardio-metabolic risk factors are implicated in the etiology of multiple non-communicable diseases such as cardiovascular diseases and cancer, with increasing prevalence of those conditions in LMICs, and accounting for increasing majority of deaths worldwide [3]. Findings of higher prevalence of overweight/ obesity, diabetes and hypertension among high-income individuals in this study highlights a critical disconnect between socio-economic resources and utilization of health care. The behavioral factors associated with cardio-metabolic risk factors eg, sedentary lifestyle, poor diet, excessive alcohol consumption, tend to increase with increasing SES, however our results suggest that higher utilization of medical care for diagnosis and treatment typically observed among high SES individuals has not yet resulted in improved cardio-metabolic health in these populations. In addition, while rural residents may be less likely to have cardio-metabolic risk factors due to healthier dietary and physical activity patterns, lack of access to care is likely more challenging compared with urban areas. Country- and region- specific interventions focused on increasing awareness of chronic diseases risk factors, promoting healthy dietary and physical activity lifestyles, and highlighting the importance of preventive health care will be important in reducing the higher burden of non-communicable diseases (NCDs) among high SES groups [35-37]. Our observation of significantly higher odds of overweight/obesity, diabetes and self-reported hypertension among men with stable-high and declining life-course SES also suggests that these interventions should begin in early life- as this may be a critical period during which adverse and protective health behaviors are developed and then sustained over the life-course. For women, the stronger associations observed with increasing life-course SES also suggests that health behaviors likely accumulate over the life-course, and intervention strategies in early life, adolescence and adulthood will likely be needed. Across these countries, concerted efforts to improve the health care delivery systems will be needed to meet future health challenges [37]. 


\section{Strengths}

This study evaluated data on a large sample of adult representative of five middle-income countries, therefore enhancing the generalizability of these results. In-person interviews and biometric measures were obtained directly by well-trained WHO field staff, enhancing the quality and internal validity of our study variables. Life-course SES was measured consistently with other studies, and the current analysis focused on maternal and individual education since these variables have been observed to be highly correlated with health behaviors in prior studies [38].

\section{Limitations}

Data on SES and certain risk factors were based on self-reported information which may be vulnerable to recall bias. Classification of participants on the risk factor measurements might underestimate the number of those with the risk factors or participants with ongoing treatment, which may bias the result towards the null. In addition, we used BMI to assess overweight/obesity although studies suggest that central obesity may be a more important measure of adiposity in certain population groups.

\section{CONCLUSIONS}

Stable high and declining life-course SES was associated with increased odds of overweight/obesity, diabetes and hypertension among men in the middle-income countries examined. Among women, stable high, increasing and declining life-course SES was also associated with increased odds of overweight/obesity. These patterns suggest that the influence of SES on adult health for men may operate during critical periods in early life, while for women, it may be accumulative over the life-course. The majority of adults who were hypertensive based on blood pressure measurements by trained interviewers were unaware of their condition, indicating an urgent need for increased awareness, primary prevention and treatment.

\footnotetext{
Acknowledgement: The authors thank the study staff and interviewers, as well as the study participants of
the WHO SAGE study.
Availability of data and material: The dataset analyzed in this study are currently available in the WHO re-
pository, http://apps.who.int/healthinfo/systems/surveydata/index.php/catalog.
Ethics approval and consent to participate: This study was considered exempt due to the use of publicly
available, de-identified data.
Funding: KO is supported for her MPH by a fellowship award from the American Association of University
Women. TA is supported by the National Institutes of Health grant KO1 TWO10271-01A1.
Author's contribution: Concept, methodology and design: TA; Acquisition of data: TA; Analysis and interpre-
tation of data: TA, DTD and KO; Writing, review, and revision of manuscript: TA, DTD and KO.
Competing interests: All authors have completed the ICMJE uniform disclosure form at http://www.icmje.org/
coi_disclosure.pdf (available upon request from the corresponding author) and declare no conflicts of interest.
}

1 Zimmet PZ, Alberti KG. Introduction: Globalization and the non-communicable disease epidemic. Obesity (Silver Spring). 2006;14:1-3. Medline:16493116 doi:10.1038/oby.2006.1

2 Maire B, Lioret S, Gartner A, Delpeuch F. Nutritional transition and non-communicable diet-related chronic diseases in developing countries. [Article in French]. Sante. 2002;12:45-55. Medline:11943638

3 Wang Y, Lim H, Wu Y. Growing global burden of chronic noncommunicable diseases and an alarming situation in China. Beijing Da Xue Xue Bao. 2012;44:688-93. Medline:23073576

4 Meetoo D. Chronic diseases: the silent global epidemic. Br J Nurs. 2008;17:1320-5. Medline:19060813 doi:10.12968/ bjon.2008.17.21.31731

5 Sarrafzadegan N, Kelishadi R, Esmaillzadeh A, Mohammadifard N, Rabiei K, Roohafza H, et al. Do lifestyle interventions work in developing countries? Findings from the Isfahan Healthy Heart Program in the Islamic Republic of Iran. Bull World Health Organ. 2009;87:39-50. Medline:19197403 doi:10.2471/BLT.07.049841

6 Milton K, Macniven R, Bauman A. Review of the epidemiological evidence for physical activity and health from low- and middle-income countries. Glob Public Health. 2014;9:369-81. Medline:24697197 doi:10.1080/17441692.2014.894548

7 Noto D, Cefalů AB, Barbagallo CM, Ganci A, Cavera G, Fayer F, et al. Baseline metabolic disturbances and the twenty-five years risk of incident cancer in a Mediterranean population. Nutr Metab Cardiovasc Dis. 2016;26:1020-5. Medline:27511705 doi:10.1016/j.numecd.2016.07.003 
8 Ball K, Mishra GD. Whose socioeconomic status influences a woman's obesity risk: her mother's, her father's, or her own? Int J Epidemiol. 2006;35:131-8. Medline:16284404 doi:10.1093/ije/dyi216

9 Senese LC, Almeida ND, Fath AK, Smith BT, Loucks EB. Associations between childhood socioeconomic position and adulthood obesity. Epidemiol Rev. 2009;31:21-51. Medline:19648176 doi:10.1093/epirev/mxp006

10 Sommer I, Griebler U, Mahlknecht P, Thaler K, Bouskill K, Gartlehner G, et al. Socioeconomic inequalities in non-communicable diseases and their risk factors: an overview of systematic reviews. BMC Public Health. 2015;15:914. Medline:26385563 doi:10.1186/s12889-015-2227-y

11 Buckland G, Salas-Salvadó J, Roure E, Bulló M, Serra-Majem L. Sociodemographic risk factors associated with metabolic syndrome in a Mediterranean population. Public Health Nutr. 2008;11:1372-8. Medline:18702840 doi:10.1017/ S1368980008003492

12 Lissner L, Wijnhoven TM, Mehlig K, Sjöberg A, Kunesova M, Yngve A, et al. Socioeconomic inequalities in childhood overweight: heterogeneity across five countries in the WHO European Childhood Obesity Surveillance Initiative (COSI2008). Int J Obes (Lond). 2016;40:796-802. Medline:27136760

13 Hosseinpoor AR, Parker LA, Tursan d'Espaignet E, Chatterji S. Socioeconomic inequality in smoking in low-income and middle-income countries: results from the World Health Survey. PLoS One. 2012;7:e42843. Medline:22952617 doi:10.1371/journal.pone.0042843

14 Montez JK, Bromberger JT, Harlow SD, Kravitz HM, Matthews KA. Life-course Socioeconomic status and metabolic syndrome among midlife women. J Gerontol B Psychol Sci Soc Sci. 2016;71:1097-107. Medline:26926957 doi:10.1093/ geronb/gbw014

15 Trivedi T, Liu J, Probst JC, Martin AB. The metabolic syndrome: are rural residents at increased risk? J Rural Health. 2013;29:188-97. Medline:23551649 doi:10.1111/j.1748-0361.2012.00422.x

16 Howlader M. A Cross-section Assessment of Mother's Health and Child Health SSRN, 2014. https://papers.ssrn.com/ sol3/papers.cfm?abstract_id=2436549.

17 Goodman J, Currie J. Parental socioeconomic status, child health, and human capital. International Encyclopedia of Education, 2:253-259, 2, 253-259. Available at: http://j.mp/2oYEG24 Accessed: 22 September 2017.

18 Pudrovska T, Logan ES, Richman A. Early-life social origins of later-life body weight: the role of socioeconomic status and health behaviors over the life course. Soc Sci Res. 2014;46:59-71. Medline:24767590 doi:10.1016/j.ssresearch.2014.02.007

19 US Census Bureau. International Data Base world population estimate for 2010. August 2017. Available: https://www. census.gov/population/international/data/idb/worldpop.php. Accessed: 13 October 2017.

20 Wang Y. Cross-national comparison of childhood obesity: the epidemic and the relationship between obesity and socioeconomic status. Int J Epidemiol. 2001;30:1129-36. Medline:11689534 doi:10.1093/ije/30.5.1129

21 Nayak SB, Rahming V, Raghunanan Y, Raghoonath C, Rahman A, Rajh D, et al. Prevalence of diabetes, obesity and dyslipidaemia in persons within high and low income groups living in North and South Trinidad. J Clin Diagn Res. 2016;10:IC08-IC13. Medline:27437244

22 De Silva AP, De Silva SH, Haniffa R, Liyanage IK, Jayasinghe KS, Katulanda P, et al. A cross sectional survey on social, cultural and economic determinants of obesity in a low middle income setting. Int J Equity Health. 2015;14:6. Medline:25595202 doi:10.1186/s12939-015-0140-8

23 Albrecht SS, Gordon-Larsen P. Socioeconomic gradients in body mass index (BMI) in US immigrants during the transition to adulthood: examining the roles of parental education and intergenerational educational mobility. J Epidemiol Community Health. 2014;68:842-8. Medline:24847088 doi:10.1136/jech-2014-203814

24 Sönmez K, Pala S, Mutlu B, Izgi A, Bakal RB, Incedere O, et al. Distribution of risk factors according to socioeconomic status in male and female cases with coronary artery disease. [Article in Turkish]. Anadolu Kardiyol Derg. 2004;4:3015. Medline: 15590357

25 Mendez MA, et al. Income, education, and blood pressure in adults in Jamaica, a middle-income developing country. Int J Epidemiol. 2003;32:400-8. Medline:12777427 doi:10.1093/ije/dyg083

26 Le C, Jun D, Zhankun S, Yichun L, Jie T. Socioeconomic differences in diabetes prevalence, awareness, and treatment in rural southwest China. Trop Med Int Health. 2011;16:1070-6. Medline:21668591 doi:10.1111/j.1365-3156.2011.02805.x

27 Wu H, Meng X, Wild SH, Gasevic D, Jackson CA. Socioeconomic status and prevalence of type 2 diabetes in mainland China, Hong Kong and Taiwan: a systematic review. J Glob Health. 2017;7:011103. Medline:28702177 doi:10.7189/ jogh.07.011103

28 Busingye D, Arabshahi S, Subasinghe AK, Evans RG, Riddell MA, Thrift AG. Do the socioeconomic and hypertension gradients in rural populations of low- and middle-income countries differ by geographical region? A systematic review and meta-analysis. Int J Epidemiol. 2014;43:1563-77. Medline:24867304 doi:10.1093/ije/dyul12

29 Quispe R, Benziger CP, Bazo-Alvarez JC, Howe LD, Checkley W, Gilman RH, et al. The relationship between socioeconomic status and CV Risk Factors: The CRONICAS Cohort Study of Peruvian Adults. Glob Heart. 2016;11:121-130.e2. Medline:27102029 doi:10.1016/j.gheart.2015.12.005

30 Lin YC, Yen LL, Chen SY, Kao MD, Tzeng MS, Huang PC, et al. Prevalence of overweight and obesity and its associated factors: findings from National Nutrition and Health Survey in Taiwan, 1993-1996. Prev Med. 2003;37:233-41. Medline:12914829 doi:10.1016/S0091-7435(03)00119-1

31 Cappuccio FP, Miller MA. Cardiovascular disease and hypertension in sub-Saharan Africa: burden, risk and interventions. Intern Emerg Med. 2016;11:299-305. Medline:27001886 doi:10.1007/s11739-016-1423-9 
32 Keenan K, Ploubidis GB, Silverwood RJ, Grundy E. Life-course partnership history and midlife health behaviours in a population-based birth cohort. J Epidemiol Community Health. 2017;71:232-8. Medline:27655423 doi:10.1136/jech2015-207051

33 Gupta R, Kaur M, Islam S, Mohan V, Mony P, Kumar R, et al. Association of household wealth index, educational status, and social capital with hypertension awareness, treatment, and control in South Asia. Am J Hypertens. 2017;30:37381. Medline:28096145

34 Link BG, Northridge ME, Phelan JC, Ganz ML. Social epidemiology and the fundamental cause concept: on the structuring of effective cancer screens by socioeconomic status. Milbank Q. 1998;76:375-402. Medline:9738168 doi:10.1111/14680009.00096

35 Vialle-Valentin CE, Serumaga B, Wagner AK, Ross-Degnan D. Evidence on access to medicines for chronic diseases from household surveys in five low- and middle-income countries. Health Policy Plan. 2015;30:1044-52. Medline:25255920 doi:10.1093/heapol/czul07

36 Kwaśniewska M, Kaleta D, Dziankowska-Zaborszczyk E, Drygas W. Healthy behaviours, lifestyle patterns and sociodemographic determinants of the metabolic syndrome. Cent Eur J Public Health. 2009;17:14-9. Medline:19418714

37 Arredondo A, Recaman AL, Azar A. Socioeconomic determinants and health disparities in relation to hypertension in middle-income countries. Am J Hypertens. 2017. [Epub ahead of print]. Medline:28199506 doi:10.1093/ajh/hpx016

38 Akinyemiju T, Ogunsina K, Okwali M, Sakhuja S, Braithwaite D. Lifecourse socioeconomic status and cancer-related risk factors: Analysis of the WHO study on global ageing and adult health (SAGE). Int J Cancer. 2017;140:777-87. Medline:27813060 doi:10.1002/ijc.30499 